\title{
LEGISLATIVE NEWS
}

\section{INFECTION CONTROL}

\section{TSS Information Required on Tampon Package Labels}

Health and Human Services Secretary Richard S. Schweiker and FDA Commissioner Arthur Hull Hayes, Jr., M.D., have announced a final regulation requiring that information about toxic shock syndrome be placed on tampon package labels.

The regulation requires either a comprehensive statement on the package or a shorter alert advising users to read an information leaflet about the disease that is included in the package.

The shorter alert reads: "ATTENTION: Tampons are associated with Toxic Shock Syndrome (TSS). TSS is a rare but serious disease that may cause death. Read and save the enclosed information."

The regulation was approved by Schweiker on June 2, 1982. Since that time consumer and professional groups had urged more information for women on TSS and tampons, such as a labeling statement, most recently at a June 4 press conference reporting on a TSS study done by the National Academy of Sciences which confirmed the continuing tampon-TSS association.

"By providing these warning labels," said Schweiker, "we will be giving women important information that can help protect them against the dangers of tampon-associated toxic shock syndrome."

Both Schweiker and Hayes praised tampon manufacturers for voluntarily including information on TSS in or on packages since 1980, when Centers for Disease Control (CDC) studies associated the disease with tampon use. Hayes added that the regulation is needed "to provide consistency in the kind of information provided."

CDC has received reports of more than 1,660 cases of TSS, including 88 deaths. About 50 TSS cases a month continue to be reported to CDC.

When FDA proposed a warning label for tampons, it received 344 comments from consumers, manufacturers, consumer and industry organizations and state, local and federal health agencies, almost all in support of some form of package information about TSS.

This regulation gives manufacturers some flexibility but requires the statements on or in the tampon package to include information on:

- The warning signs of TSS sudden high fever (usually $102^{\circ} \mathrm{F}$ or more) and vomiting or diarrhea. (Other signs may include fainting or nearly fainting when standing up, dizziness and a rash that looks like sunburn.)

- What to do if these signs appear remove the tampon at once and seek medical help right away.

- The incidence of TSS in women, and the higher risk among those under 30 and teen-age girls, and the risk of death.

- The advisability of using tampons with the least absorbency needed. (One study has indicated that the risk of TSS appears to be smaller with less absorbent tampons.)

- The avoidance of the risk of tampon-related TSS by not using tampons, or possibly reducing the risk by alternating tampons with sanitary napkins during menstrual periods.

\section{Hatch Amendments Clarify Medicare Reimbursement to Hospitals}

Amendments proposed by Senator
Orrin Hatch (R-Utah) clarify Medicare reimbursement to hospitals experiencing changes in the types of medical cases handled and strengthen the exemptions process whereby a hospital may request more appropriate reimbursements in certain cases.

Hatch's proposals amend H.R. 4961, the Tax Equity and Fiscal Responsibility Act of 1982 . Hospitals receive, under present law, Medicare reimbursements based on "reasonable costs" incurred in providing medical care to Medicare recipients. H.R. 4961 amends the current law by establishing a three-year, reimbursement cap de. signed to limit annual rates of medical care increases.

"The current proposal in H.R. 4961 places an expenditure limit on the hospitals per case - all of the hospital's cases are added together regardless of the type of case treated. An average cost per case is determined, and the expenditure limit is applied to this average cost per case. In other words, the costs for appendectomies and open heart surgeries are mixed together to determine the hospital's overall average cost per case," Hatch said.

"In order to keep the average cost per case down, the hospital may have an incentive to treat more routine cases and fewer complex cases. Hospitals which, over time, may be treating more elderly, more cancer patients, more open heart cases, and more intensive cases of all kinds, all else being equal, could be unintentionally jeopardized by the current proposal," Hatch said.

One of Hatch's amendments, adopted by the Senate by voice vote, strengthens the exemptions process under the three-year Medicare hospital

(continued on page 188 ) 

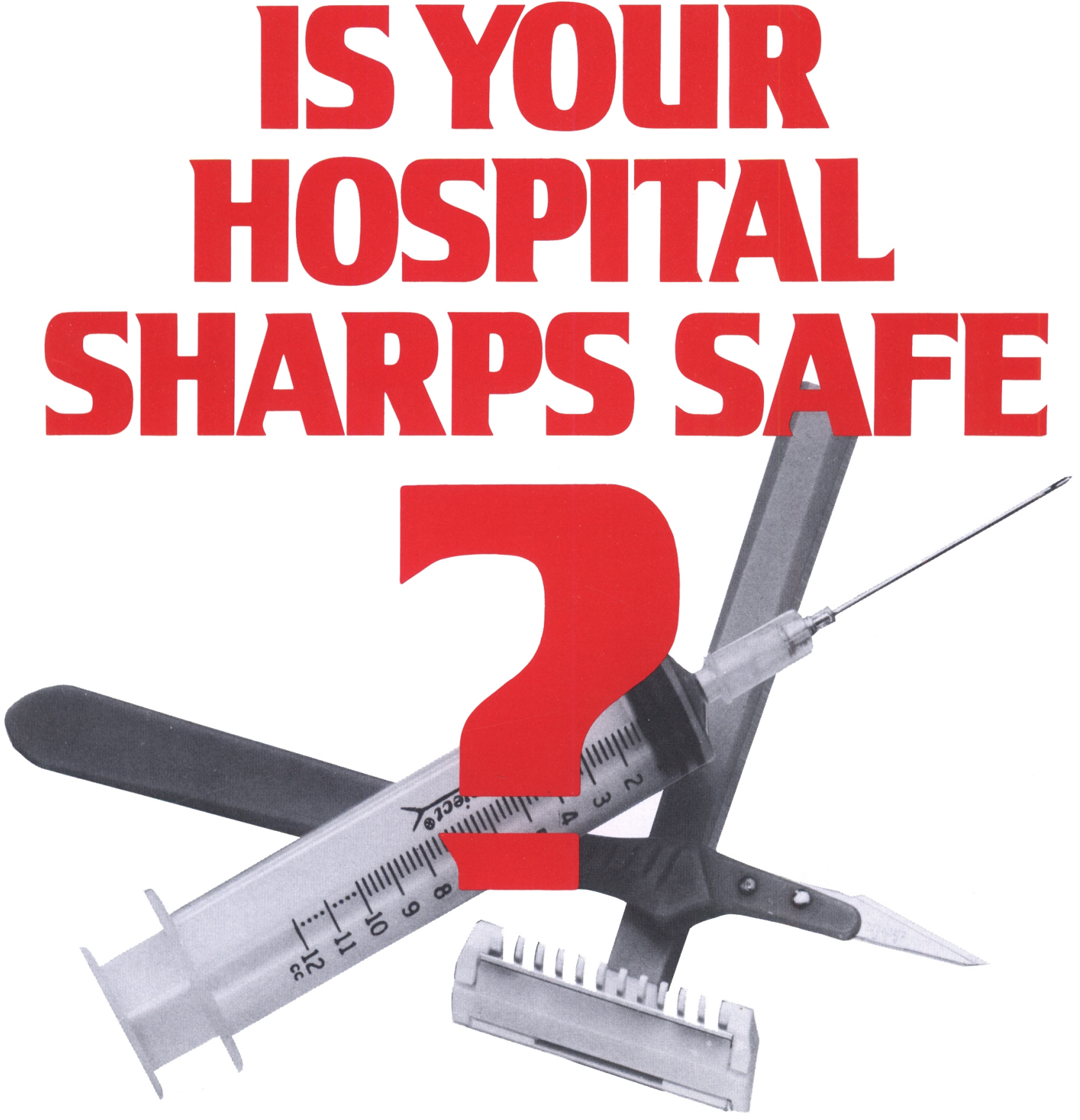

Needle sticks and other puncture wounds are dangerous to your people and expensive for your hospital. Return the coupon and we will tell you how your dirty, dangerous used sharps can be truly "untouchables" from use to refuse.

\section{MONDJECT}

\section{Mail This Coupon Today! \\ LET'S TALK ABOUT CONTROL OF SPECIAL WASTES}

Tell me how the Sharps Disposal System will costeffectively help me toward complete control of special wastes in my hospital.

NAME

TITLE

INSTITUTION

ADDRESS

CITY STATE ZIP

PHONE EXT. 


\section{(continued from page 486)}

reimbursement cap. The amendment assures that the exemption and adjustment process for the three-year rate of increase limitation provision is carried out in a manner sensitive to legitimate changes in hospital costs resulting from various circumstances - including more intensive use of the facility.

Hatch's second amendment clarifies the bill by applying the expenditure limit to the average cost of comparable cases by a "diagnostic category." Hatch, as planned, withdrew the amendment as part of a colloquy with Senator Robert Dole (R-Ka.), Chairman of the Senate Finance Committee. Hatch received assurance that the Finance Committee intended "that the Secretary of Health and Human Services establish written criteria to be used in making adjustments for case mix. Thus, a hospital will be able to utilize this criteria in assessing its position and preparing documentation to support its request for an adjustment."

\section{Senate Hearings Told Vaccine Research Can Bring Down Medical Care Costs}

"The cost to a youngster for an hour's play on one of the new coinoperated video games can be higher than Lederle Laboratories' average price for vaccines which provide lifelong immunization against four child killer diseases," according to Lederle's president at a Senate hearing.

Mr. Jack Bowman, president of Lederle Laboratories, Division of American Cyanamid Company, provided vaccine price data showing that the cost of medical care in general has far outstripped Lederle's vaccine prices over the past 20 years.

The information was presented in a statement prepared for a hearing held before the Subcommittee on Investigations and General Oversight of the Senate Committee on Labor and Human Resources.

His statement pointed out that Lederle's current average selling price for the required three doses of Orimune oral polio vaccines is about $\$ 5.00$. The average price for the required four doses of Tri-Immunol (DPT - vaccine against diphtheria, pertussis and tetanus) is $\$ 1.82$.

Since the introduction of oral polio vaccine in 1963, Lederle's average selling price increase for Orimune has been almost $100 \%$ lower than the $244 \%$ medical care cost increase for the same 19 -year period. The price increase for Lederle's DPT vaccine from 1962 to 1982 was held to $159 \%$ against a medical care cost increase of $252 \%$ over the same period.

Mr. Bowman expressed concern that while seven manufacturers hold US licenses for the production of DPT vaccine, only three presently remain in the market. During the past decade two of the three US companies that manufactured oral polio vaccine have chosen to leave that market.

"That rising costs and inadequate prices have been a significant factor in the decisions to withdraw from vaccine markets," Mr. Bowman stated, "is attested to by the conclusions of a government study, Reports and Recommendations of the National Immunization Work Groups, initiated by the Office of the US Assistant Secretary for Health and published in 1977."
The study concludes: "While the potential capability of American in. dustry to supply vaccine requirements is unarguable, there has been a steady attrition of specific pharmaceutical manufacturers from the entire field of biologics. A relatively low profit margin, high production risks, increasing costs of research and development, difficulties in clinical testing, and increasingly stringent governmental standards of safety and efficacy are all formidable constraints to private investment."

As evidence of Lederle's intention of remaining in the vaccine market, Mr. Bowman's statement referred to several research and development programs Lederle now has underway. These include a vaccine for haemophilus influenzae, identified as a high priority project by the National Institutes of Health. This disease is the main cause of acute bacterial meningitis which strikes young children and causes the deaths of ten percent of those affected. It is expected that the research and development will cost at least $\$ 50$ million. Also being undertaken is the development of a vaccine against the herpes virus which will cost at least $\$ 30$ million. As part of Lederle's ongoing product improve. ment programs, research is underway to develop an a-cellular pertussis vaccine.

"It is Lederle's expectation and hope," Mr. Bowman's statement concluded, "that we will be able to bring these efforts to a successful conclusion inasmuch as effective immunizing agents not only save lives and prevent illness, but can be a significant force in reducing this nation's growing bill for medical care." 

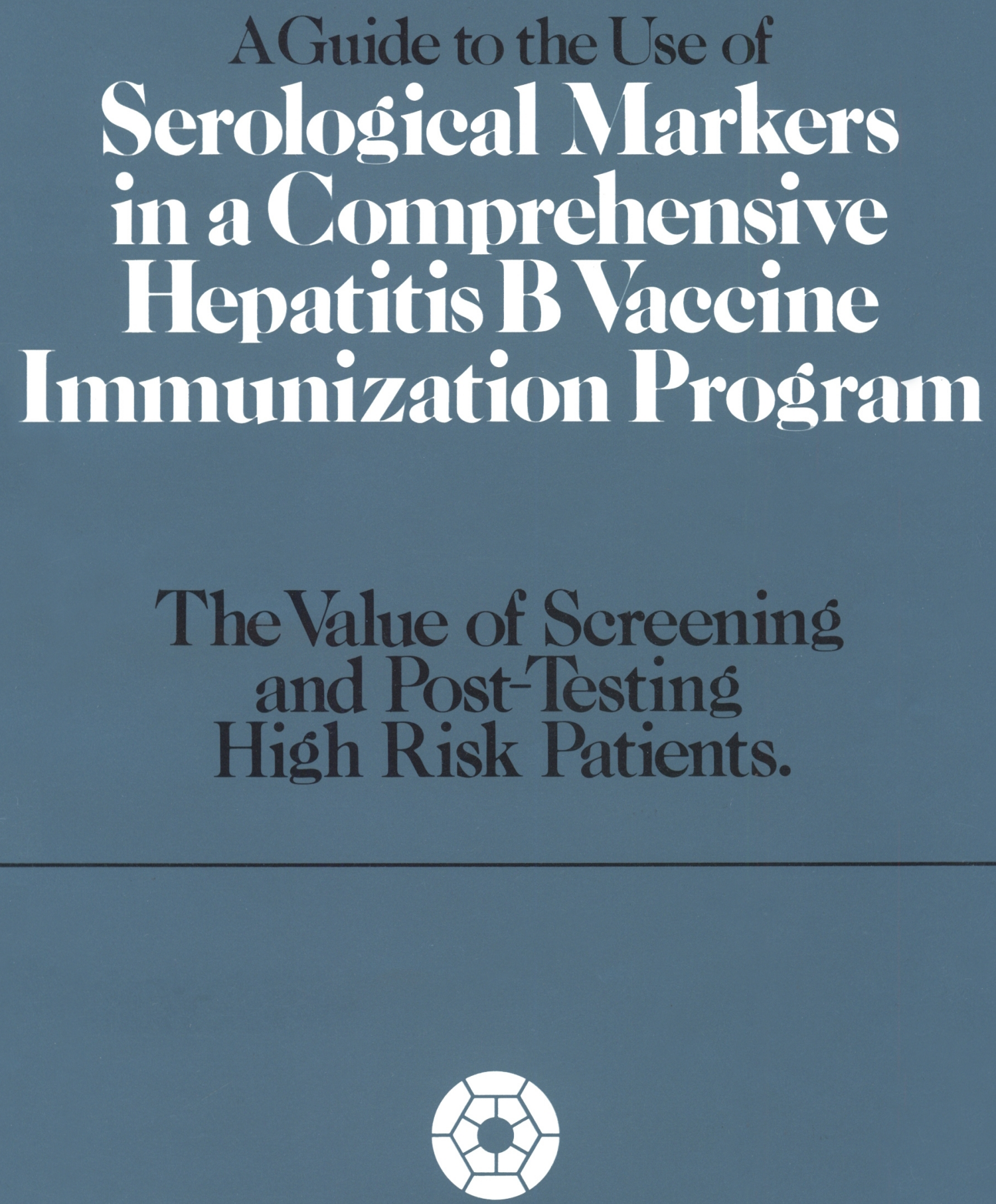

HEPATITIS INFORMATION CENTER

Abbott Laboratories, Diagnostics Division 


\section{Assessing the Need...}

A significant advance in immunology.

The introduction of Heptavax- $B^{\circledast}$ (Hepatitis B Vaccine, MSD)

holds the promise of effecting a marked reduction in the

incidence of hepatitis B virus (HBV) infection.

Who should receive the vaccine?

Certain demographics will identify those groups which should have priority access to the vaccine. Recognized high risk categories include: medical and dental personnel, hemodialysis patients and the staff at dialysis centers, laboratory personnel, homosexually active males, institutionalized patients, paramedics, close contacts of chronic carriers, travelers to and immigrants from endemic areas, and illicit drug users.

Why screen high risk candidates for anti-HBs and anti-HBC prior to vaccination?

The supply and cost of hepatitis B vaccine plus the fact that a percentage of the high risk groups cannot benefit from the vaccine (due to previous or current HBV infection) make it clear that individual need should be established. Giving it to those who cannot benefit is a waste of resources and finances.

Testing the patient for anti-HBs and anti-HBc provides the physician with the relevant data. The procedure is uncomplicated and cost effective.

A positive test result for anti-HBs (antibody to hepatitis B surface antigen) indicates clinical recovery from and immunity to hepatitis $B$ infection.

A positive anti-HBc (antibody to hepatitis B core antigen) test result is an early indication of acute infection. It is also a longlasting marker that can be detected for many years and is usually present long after recovery from acute hepatitis.

By ordering both tests, it is unlikely that any subjects with asymptomatic acute or chronic infection, or previous exposure, would be missed (as is possible with the anti-HBs test only). Also, the high risk patient who is only positive for anti-HBs would not be missed. Furthermore, some individuals may test anti-HBs negative/anti-HBc positive. These should be tested for HBsAg (surface antigen). If this marker is positive, an acute or chronic infection is confirmed; vaccination is not necessary. The value of post-testing for anti-HBs.

There has been discussion of the validity of post-testing for anti-HBs to verify immunity. Primarily, this has been based on the clinical findings of two studies. In one, there was evidence of immunity in 96 percent of the subjects after administration of the 3-dose regimen! In the other, 85 percent attained immunity?

There is a correlation between age and responsiveness, and between the person's general health and responsiveness. Adults do not respond as vigorously as do children. Healthy individuals respond better than people who are immunosuppressed or immunocompromised?

While the studies support the efficacy of the vaccine, these findings do not obviate the need to know if immunity definitely exists subsequent to vaccination and if the immunity continues to exist.

In most cases where serologic evidence indicates that the individual can benefit, the vaccine will induce the development of anti-HBs. Immunity will be achieved. For how long, is not known. Periodic testing for anti-HBs will confirm the presence of immunity over time.

The use of serological markers is important to an effective hepatitis $B$ immunization program.

A screening program will help assure that the available supply goes only to those who can benefit; specifically, individuals who do not express serologic evidence of previous or current exposure. The post-test program should be used because of the obvious consequences that can arise if the recipient who assumes immunity subsequently becomes infected.

\section{REFERENCES}

1. Szmuness, W.; Stevens, C.E.; Harley, E.J., et al: Hepatitis B vaccine: Demonstration of efficacy in a controlled clinical trial in a high-risk population in the United States, N. Engl. J. Med. 303 (15): 833-841, October 9, 1980.

2. Heptavax- $B^{\circledast}$ (Hepatitis $B$ vaccine, MSD), package insert issued by Merck Sharp \& Dohme, May 1982, A.H.F.S. Category: 80:12.

3. Hillerman, M.R.; Buynak, E.B.; McAleer, W.J., et al: Hepatitis A and hepatitis B vaccines, in Viral Hepatitis 1981, W. Szmuness; H. Alter, J. Maynard (ed.), Philadelphia, The Franklin Inst. Press, 1982.

\section{Hepatitis Management: Understanding and Monitoring Viral Hepatitis B}

CUT ALONG DOTTED LINE

\section{HEPATITIS INFORMATION CENTER \\ Abbott Laboratories \\ Diagnostics Division AP6C4 \\ North Chicago, IL 60064 \\ Please reserve my free copy}

\section{Hepatitis Management:}

Understanding and Monitoring Viral Hepatitis B
An invaluable addition to your reference shelf... a practical tool to help you identify and test hepatitis $B$ vaccine candidates, and diagnose and manage patients with hepatitis. You'll receive:

- The Hepatitis Forum issue dedicated to the hepatitis B vaccine.

- Definitive monographs covering all types, all aspects of viral hepatitis.

- Hepatitis algorithm detailing the recommended steps in serodiagnostic assessment.

This kit will be available in limited quantities. Reserve your free copy now by sending coupon or writing to...

\section{HEPATITIS INFORMATION CENTER}

Abbott Laboratories

Diagnostics Division AP6C4

North Chicago, IL 60064

ABBOTT LABORATORIES

Diagnostics Division North Chicago, IL 60064 